\title{
Aerosol box for peripheral nerve blocks
}

\author{
Aleksandar Dimitrovski, Marija Toleska, Andrijan Kartalov, Biljana Kuzmanovska \\ University Clinic for TOARILUC-KARIL, Medical Faculty Skopje, University "Ss Cyril and Methodius" Skopje, Macedonia
}

Correspondence: Aleksandar Dimitrovski, MD, Todor Aleksandrov 73-1/21 1000 Skopje, North Macedonia; E-mail: dimitrovski777@gmail.com; Phone: +389 70250692

Citation: Dimitrovski A, Toleska M, Kartalov A, Kuzmanovska B. Aerosol box for peripheral nerve blocks. Anaesth. pain intensive care 2020;24(4):469-470. DOI: $10.35975 /$ apic.v24i4.1323

The COVID-19 pandemic has posed new challenges to the anesthesiologists the world over. Keeping in mind the way the virus was known to be spreading through aerosols and the global deficiency of personal protective equipment, medical workers embarked upon to create and develop devices and equipment to protect themselves from the virus during this pandemic period.

Taiwanese anesthesiologist Dr. Hsien Yung Lai has constructed an aerosol box, ${ }^{1}$ whose function is to offer an additional protection from aerosols produced during the induction and intubation of the patients needing surgery. The box is transparent made of plexiglass with dimensions of $50 \times 50 \times 40 \mathrm{~cm}$. The box has two holes with diameters of $10 \mathrm{~cm}$ each, for insertion and manipulation of the hands of the intubating anesthesiologist.

Taking consideration of the recommendations for COVID-19 by The European Society of Regional Anaesthesia \& Pain Therapy (ESRA) and The American Society of Regional Anesthesia and Pain Medicine (ASRA). ${ }^{2}$ the preference is given to peripheral nerve blocks as a main anesthesia technique, thus avoiding the need of intubation and general anesthesia.

We used the aerosol box as a protective shield for performing peripheral nerve blocks of brachial plexus, by infraclavicular and interscalene approach and cervical plexus block in patients who were suspected of CoViD-19 infection. Many studies concluded that the potential aerosol spread of SARSCoV-2 is not only through coughing and sneezing, but also through breathing. ${ }^{3}$ In a large number of these patients, a cough is a symptom which represents a high possibility of transmitting the virus through aerosols

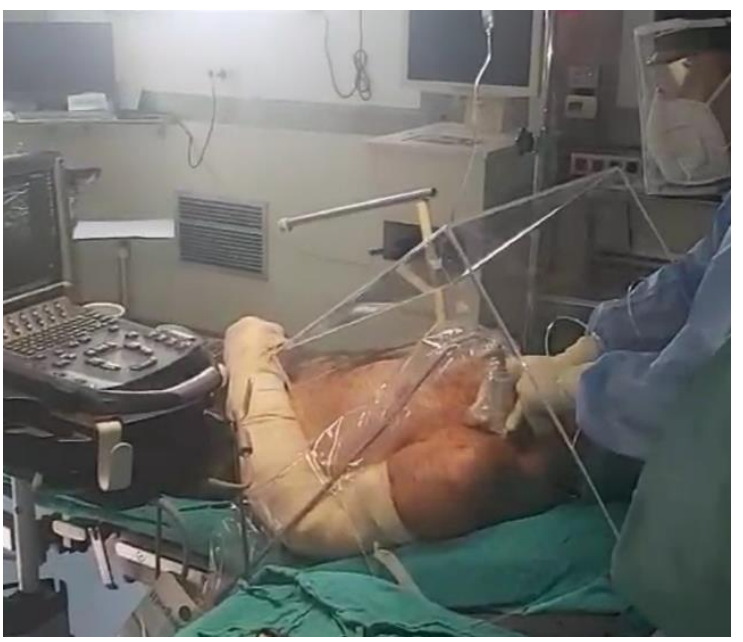

Figure 1: Performing US guided infraclavicular nerve block with aerosol box

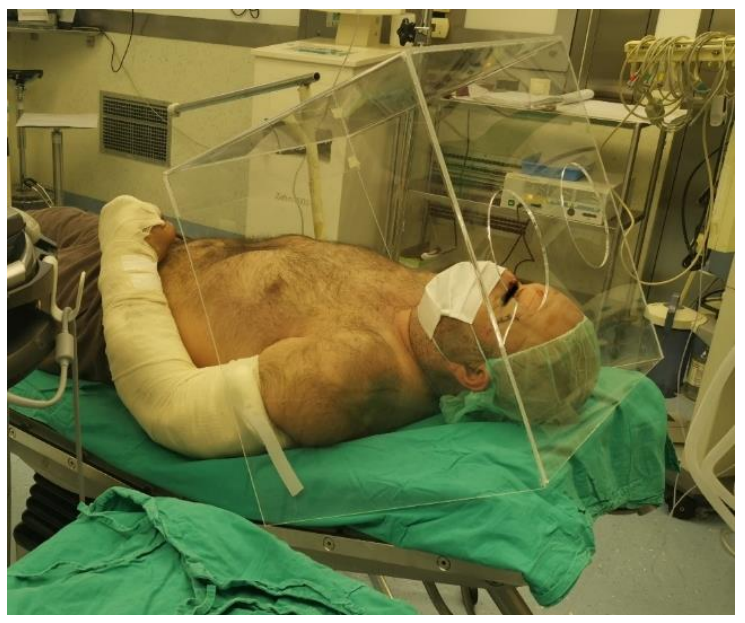

Figure 2: Positioning of the aerosol box on patient

to the anesthesiologist, because of the close contact that they have during the performance of these blocks.

The box is positioned over the patient's head. Through the one hole, the anesthesiologist inserts one hand with the needle for applying the peripheral nerve block; 
through the other hole, the other hand holds the probe of the ultrasound. During the procedure, the anesthesiologist is using the recommended PPE.

Although the space is limited for manipulation, we successfully performed the blocks without much limitations.

We think that the use of aerosol box during manipulation and performing the peripheral nerve blocks in brachial and cervical region, in patients that are suspected of COVID-19, or are known to be infected, enhances the safety of the anesthesiologist as the chances of infection during the manipulation are extremely low.

\section{References}

1. Everington K. Taiwanese doctor invents device to protect US doctors against coronavirus. Taiwan News. March 23, 2020 [Internat\}; Available from: https://www.taiwannews.com.tw/en/news/39024359. Accessed on 17 July 2020)

2. Uppal V, Sondekoppam RV, Lobo CA, Kolli S, Kalagara HKP. Practice Recommendations on Neuraxial Anesthesia and Peripheral Blocks during the COVID-19 Pandemic [Internet]; Available on https://www.asra.com/covid-19/raguidance (Accessed 10 July 2020)

3. National Academies of Sciences, Engineering, and Medicine. 2020. Rapid Expert Consultation on SARS-CoV-2 Viral Shedding and Antibody Response for the COVID-19 Pandemic (April 8, 2020). Washington, DC: The National Academies Press. DOI: 10.17226/25774

\title{
USG for assessing nostril patency for successful nasotracheal intubation
}

\author{
Teena Bansal, Jatin Lal \\ Department of Anaesthesiology \& Critical Care \\ Pt. B.D. Sharma University of Health Sciences, Rohtak-124001 (Haryana), India.
}

Correspondence: Dr. Teena Bansal, 19/6 J, Medical Campus PGIMS, Rohtak-124001 (Haryana), India; E-mail: aggarwalteenu@rediffmail.com; Mobile: +91-9034239374

Citation: Bansal T, Lal J. USG for assessing nostril patency for successful nasotracheal intubation. Anaesth. pain intensive care 2020;24(4):470-471. DOI: 10.35975/apic.v24i4.1324

A 45-year male was scheduled for reduction of fracture mandible. Mouth opening was $1 \mathrm{~cm}$. So fibreoptic nasotracheal intubation was planned. Xylometazoline drops were instilled in the nostrils. Right nostril was more patent as compared to the left one. So a split nasopharyngeal airway size $7 \mathrm{~mm}$ was tried to be introduced through this nostril. But the attempt failed, so it was removed and blood was found on it reflecting trauma. Nasal drops were instilled in right nostril and then the same nasopharyngeal airway was introduced through the left nostril. Its insertion was very smooth and nasotracheal intubation was done successfully. Postoperatively ultrasound of nasal cavity was carried out and left nostril was found to be more patent contrary to preoperative examination.

Nasotracheal intubation is routinely performed procedure for maxillofacial surgeries. Patency of nostril is an important factor for successful intubation in addition to prevent intubation related trauma. Several tests have been introduced to assess the suitable nostril for nasotracheal intubation. Two simple tests are occlusion test and spatula test. But both these tests have a diagnostic failure rate of approximately $45 \%$ when an abnormal nostril was present. Rhinomanometry evaluates the patency of nostrils but it is not simple and requires special equipment. So, this method is not commonly used in clinical practice. ${ }^{1,2}$

Hence, we should not rely on occlusion test for determining nasal patency. Ultrasound of nasal cavity is suggested to confirm patency of nostril for nasotracheal intubation to increase success and prevent complications. 


\section{References}

1. Smith JE, Reid AP. Identifying the more patent nostril before nasotracheal intubation. Anaesthesia. 2001;56:258-262. [PubMed] DOI: 10.1046/.13652044.2001.01717-3.x
2. Shohara K, Goto T, Kuwahara G, Isakari Y, Moriya T, Yamamuro T. Validity of rhinometry in measuring nasal patency for nasotracheal intubation. J Anesth. 2017;31:1-4. [PubMed] DOI: 10.1007/s00540-016$\underline{2262-6}$

\title{
CORRESPONDENCE
}

CORONA EXPERIENCE

\section{The alarming rate of COVID-19 among pregnant women in Pakistan}

\author{
Rooh Ullah', Fawad Shabir Memon², Samiaa Anjum ${ }^{3}$ \\ 1-Department of Microbiology, Quaid-i-Azam University, Islamabad, Pakistan. \\ 2-National Institute of Health, Islamabad, Pakistan. Shaheed Zulfiqar Ali Bhutto Institute of Science and Technology (SZABIST), Karachi, Pakistan. \\ 3-Department of Microbiology, Quaid-i-Azam University, Islamabad, Pakistan. \\ Correspondence: Rooh Ullah, M Phil, Department of Microbiology, Quaid-i-Azam University, Islamabad, Pakistan; E-mail: \\ roohullah@bs.qau.edu.pk
}

Citation: Ullah R, Memon FS, Anjum S. The alarming rate of COVID-19 among pregnant women in Pakistan. Anaesth. pain intensive care 2020;24(4):471-472. DOI: $\underline{10.35975 / a p i c . v 24 i 4.1325}$

In the previous two months, a rapid increase in the number of COVID-19 patients has been observed in various cities of Pakistan such as Gujrat, Lahore and Karachi. The pandemic exhibits a peculiar challenge to the obstetric community, as most of them have to be admitted to a hospital for normal or cesarean delivery. There link with the medical care system starts right from the start with the much needed antenatal visits. On 15th March 2020, first pregnant woman was diagnosed with COVID-19 in Pakistan. ${ }^{1}$ This was followed by the implementation of universal testing protocol including nasopharyngeal swabs and quantitative polymerase-chain reaction (q-PCR) for the sole purpose to detect the SARS-CoV-2 infection in obstetric women admitted or in need of admission to a hospital for childbirth.

From 29th March to 9th May 2020, 303 deliveries were conducted at the Government Maternity Hospital (GMH) Gujrat, Basic Health Unit (BHU) Fatehpur, Shaikh Zayed Hospital Lahore and DUHS Hospital Karachi. At the time of admission to the hospitals, COVID-19 symptoms were screened in all the pregnant women and it was reported that raised temperature, along with many different symptoms, was observed in 98 (32.3\%) of them. Out of these 98 women, COVID-19 was positively detected in 28 (28.5\%) pregnant women.
The rest of the $205(67.6 \%)$ women did not reveal a raised temperature or any of the symptoms at the admission time. Out of these 205 asymptomatic parturients, $3(1.4 \%)$ were detected to be positive for the COVID-19. Hence, of the total 303 patients, 31 $(10.2 \%)$ obstetric women were screened positive.

Out of the three women who did not have any symptom of the disease, but were still positive for the infection at the time of admission, only one patient developed fever before her discharge from the hospital after the delivery ( 2 days are the average length of stay after the childbirth). Antibiotics were received by one of the other two parturients, for the treatment of suspected endometritis. The patient, who was assumed to be febrile due to COVID-19, received supportive treatment.

The implementation of testing protocols for the detection of the virus in the pregnant population at the time of admission to the hospitals for delivery indicated that in Pakistan, almost 33\% parturients displayed various symptoms of the disease. Although this finding cannot be generalized to geographic areas with lesser infection rates, it emphasizes the COVID19 risks amongst symptomatic pregnant patients. ${ }^{2} \mathrm{~A}$ limited availability of data from whole of the country along with false negative test results, might lead us to underestimate the exact prevalence of the infection; 
but still the COVID-19 prevalence among the pregnant women seems to be alarming. ${ }^{3}$

The use of testing practices at the admission time provides the possible advantage allowing us to reassess hospital isolation procedures, pre-empt the bed assignments, to notify newborns service centers and give guidance about the usage of personal protective materials. Collection of similar clinical data is needed to ensure safety of the infants, mothers, as well as healthcare workers during this difficult period of pandemic.
1. Javaid S, Javaid MK. Survey on corona virus: a case study in Pakistan. International J Med Sci Clin Res Rev. 2020 Apr 17;3(02,):223-227. [Free Full Text]

2. Sutton D, Fuchs K, D'alton M, Goffman D. Universal screening for SARS-CoV-2 in women admitted for delivery. N Eng J Med. 2020 May 28;382(22):21632216. [PubMed] DOI: $10.1056 / N E J M c 2009316$

3. Ai T, Yang Z, Hou H, Zhan C, Chen C, Lv W, et al. Correlation of chest CT and RT-PCR testing for coronavirus disease 2019 (COVID-19) in China: A report of 1014 cases. Radiology. 2020 Aug;296(2):E32E40. [PubMed] DOI: $10.1148 /$ radiol.2020200642

\section{References}

\section{CORRESPONDENCE}

CORONA EXPERIENCE

\section{Lignocaine use in respiratory viruses}

\section{Amjid Hammodi}

Orthopedic Surgeon, Baghdad, Iraq; E-mail: amjidhammodi@yahoo.com

Citation: Hammodi A. Lignocaine use in respiratory viruses. Anaesth. pain and intensive care 2020;24(4);472.

DOI: $10.35975 / a p i c . v 24 i 4.1332$

Following the publication of the article entitled, 'Lignocaine's substantial role in COVID-19 management: potential remedial and therapeutic implications', authored by Nasser Ali Malik, Amjid Hammodi and Dayanidhi Jaiswara, published in the Anaesthesia, Pain and Intensive Care; 21(1);59-63, 2020 , I would like to suggest a revolutionary idea that could help control the spread of respiratory viruses from person to person, achieve potential prophylaxis against them and even could be used as a treatment for mild to moderately infected cases.

The idea is to use intranasal lignocaine gel to strengthen the sodium channels of the nasal and nasopharyngeal mucosal cells, as it has emerged recently that targeting ion channels of the host cells can impede virus life cycle. Theoretically, it can produce prophylaxis and also can be used as a treatment. The lignocaine gel can be applied to the nasal septum and lateral walls. The lignocaine will diffuse through the mucosa to the myelin sheaths of the nasopalatine nerve (branch of the maxillary nerve) and nasociliary nerve (branch of the ophthalmic nerve), and to the myelin sheaths of the maxillary and vagus nerves. It will eventually strengthen the nasopharyngeal mucosal cell sodium channels along with nasal mucosal cells. The theory of the ability of lipid soluble lignocaine to travel through the uninterrupted myelin sheaths of different nerves, as myelin sheaths are mainly formed off lipids themselves, has been adopted by the author as a hypothesis to explain the ability of a distal nerve block to relieve pain caused by a more proximal cause (the anatomical gates of sodium channels blockers). Thus, for prophylaxis against viral respiratory infections, applying the lignocaine gel to the nasal cavity twice daily will theoretically suffice; while infected cases can be treated with more frequent applications of the gel, up to every hour and then reducing the frequency gradually as patient's symptoms improve.

This prophecy needs to undergo clinical trials to prove or refute the benefits.

\section{Reference:}

Malik NA, Lignocaine's substantial role in COVID-19 management: potential remedial and therapeutic implications. Anaesth. pain \& intensive care 2019;23(1):84-91 [Free full text] DOI: $\underline{10.35975 / a p i c . v 24 i 1.122}$ 